\title{
Type B plactic relations for $r$-domino tableaux
}

\author{
Müge Taşkın | \\ Bogaziçi University, Turkey.
}

The recent work of Bonnafé et al. (2007) shows through two conjectures that $r$-domino tableaux have an important role in Kazhdan-Lusztig theory of type $B$ with unequal parameters. In this paper we provide plactic relations on signed permutations which determine whether given two signed permutations have the same insertion $r$-domino tableaux in Garfinkle's algorithm (1990). Moreover, we show that a particular extension of these relations can describe Garfinkle's equivalence relation on $r$-domino tableaux which is given through the notion of open cycles. With these results we enunciate the conjectures of Bonnafé et al. and provide necessary tool for their proofs.

Keywords: insertion algorithm, domino tableaux, plactic relations

\section{Introduction}

Let $W$ be a finite Coxeter group and let $L: W \mapsto \mathbb{Z}_{\geq 0}$ be a weight function such that

$$
L(u w)=L(u)+L(w) \text { if and only if } l(u w)=l(u)+l(w)
$$

where $l: W \mapsto \mathbb{Z}_{\geq 0}$ is the usual length function on $W$. As it is described by Lusztig in (15) every weight function determines an Iwahori-Hecke algebra and some preorders on $W$ whose equivalence classes are called left, right and two-sided cells. The importance of these cells lies in the fact that they carry representations of $W$ and its corresponding Iwahori-Hecke algebra $\mathcal{H}$. Furthermore they have an important role in the representation theory of reductive algebraic groups over finite or $p$-adic fields (15) and in the study of rational Cherednik algebras ( $\underline{8})$ and the Calogero-Moser spaces (9).

The case $L=l$ is in fact first introduced by Kazhdan and Lusztig in (11) as a purely combinatorial tool for the theory of primitive ideals in the universal enveloping algebras of semisimple complex Lie algebras. In this case the combinatorial characterizations of cells are well known, where Knuth (or plactic) relations appear as the mediating tool. Namely, when $W$ is type $A$ then each right (left) cell corresponds to the plactic (respectively coplactic) class of some standard Young tableau, whereas each two-sided cell consists of those permutations which lie in the plactic classes of tableaux of the same shape. This characterizations depend on Joseph's classification of primitive ideals in type A, where Knuth (plactic) relations play a crucial role.

In the types B,C and D, on the other hand the emerging combinatorial objects are standard domino tableaux. The connection is first revealed in the work of Barbash and Vogan (1) where they provide

†This work is part of my postdoctoral study at York University and Fields Institute, Toronto, Canada. 
necessary conditions for the characterizations of primitive ideals through an algorithm which uses the palindrome representations of signed permutations in order to assign to every signed permutation $\alpha$ a pair of same shape standard $r$-domino tableaux $\left(P^{r}(\alpha), Q^{r}(\alpha)\right)$ bijectively, for $r=0$ or $r=1$. Meanwhile, an analog of Knuth relations provided by Joseph in (10) established the sufficient conditions. On the other hand Garfinkle (4, 5, 6) finalized classification problem for these types by showing through her two algorithms on domino tableaux that these two sets of relations are in fact equivalent. Her first algorithm assigns any signed permutation to a pair of same shape standard $r$-domino tableaux for $r$ equals to 0 or 1 and the second defines an equivalence relation between domino tableaux through the notion of open cycles. We remark that the extension of Garfinkle and Barbash-Vogan algorithm for larger $r$ is given in (14) and (3) respectively.

The case $L \neq l$ is also known as unequal parameters Kazhdan-Lusztig theory and it appears for the types $B_{n}, I_{2}(n)$ and $F_{4}$, where the classification problem for the latter two can be dealt with computational methods, see (7). For type $B_{n}$, the weight function is determined by two integers $a, b>0$ such that $L\left(s_{i}\right)=a$ if $1 \leq i \leq n-1$ and it is equal to $b$ if $i=0$ where $s_{0}$ is the transposition $(-1,1)$ and $\left\{s_{i}=(i, i+1) \mid 1 \leq i \leq n-1\right\}$ are the type $A$ generators of $B_{n}$. Recently, the role of $r$-domino tableaux in this theory is revealed in the work of Bonnafé, Geck, Iancu, and Lam (3) through two main conjectures:

- Conjecture A: If $r a<b<(r+1) a$ for some $r \geq 0$ then two signed permutations lie in the same Kazhdan Lusztig right (left) cell if and only if their insertion (recording) $r$-domino tableau are the same.

- Conjecture B: If $b=r a$ for some $r \geq 1$ then two signed permutations lie in the same Kazhdan Lusztig right (left) cell if and only if their insertion (recording) $r$-domino tableau or $(r-1)$-domino tableau are the same.

On the other hand, in order to establish the proofs of these conjecture one definitely needs the plactic relations between signed permutations which determines when the insertion $r$-domino tableaux of two signed permutations are the same. Our aim here is to fill this gap.

Definition 1.1 For $\alpha=\alpha_{1} \ldots \alpha_{n} \in B_{n}$ and $r \geq 0$ consider the following relations:

$\mathrm{D}_{1}^{r}:$ If $\alpha_{i}<\alpha_{i+2}<\alpha_{i+1}$ or $\alpha_{i}<\alpha_{i-1}<\alpha_{i+1}$ for some $i$, then

$$
\alpha=\alpha_{1} \ldots \alpha_{i-1}\left(\alpha_{i} \alpha_{i+1}\right) \alpha_{i+2} \ldots \alpha_{n} \sim \alpha_{1} \ldots \alpha_{i-1}\left(\alpha_{i+1} \alpha_{i}\right) \alpha_{i+2} \ldots \alpha_{n}
$$

$\mathrm{D}_{2}^{r}$ : If there exists $0<j \leq r$ such that $\alpha_{j}>0$ and $\alpha_{j+1}<0$ (or $\alpha_{j}<0$ and $\alpha_{j+1}>0$ ) and $\alpha_{1} \ldots \alpha_{j} \alpha_{j+1}$ is a shuffle of some positive decreasing and negative increasing sequence ending with $\alpha_{j}$ and $\alpha_{j+1}$ (or respectively $\alpha_{j+1}$ and $\alpha_{j}$ ) then

$$
\alpha=\alpha_{1} \ldots\left(\alpha_{j} \alpha_{j+1}\right) \ldots \alpha_{r+2} \ldots \alpha_{n} \sim \alpha_{1} \ldots\left(\alpha_{j+1} \alpha_{j}\right) \ldots \alpha_{r+2} \ldots \alpha_{n}
$$

$\mathrm{D}_{3}^{r}$ : If $\left|\alpha_{1}\right|>\left|\alpha_{i}\right|$ for all $2 \leq i \leq r+2$ and $\alpha_{2} \ldots \alpha_{r+2}$ is a shuffle of some positive decreasing and negative increasing sequences, then

$$
\alpha=\alpha_{1} \alpha_{2} \ldots \alpha_{r+2} \ldots \alpha_{n} \sim\left(-\alpha_{1}\right) \alpha_{2} \ldots \alpha_{r+2} \ldots \alpha_{n}
$$

Now we are ready to state our results. 
Theorem $1.2 \alpha$ and $\beta$ in $B_{n}$ are equivalent through a series of $\mathrm{D}_{1}^{r}, \mathrm{D}_{2}^{r}$ or $\mathrm{D}_{3}^{r}$ relations if and only if they have the same insertion $r$-domino tableaux.

Remark 1.3 A set of relations for $r$-domino tableaux is defined in (3), but as it is already discussed there it is far from being sufficient for the characterization. In fact the plactic relation in (3. Section 3.8) can be shown to be equivalent to the one given with $\mathrm{D}_{1}^{r}$ and $\mathrm{D}_{2}^{r}$ here. Recently T. Pietraho has independently found another set of generators (17). Finally depending on his result and earlier version (20) of this paper, Bonnafé proved that $r$-plactic and r-cycle equivalence are sufficient for Conjecture A and B respectively, see (2).

Remark 1.4 Recall that for a signed permutation $\alpha=\alpha_{1} \ldots \alpha_{n}$ in $B_{n}$, its palindrome representation is given by $\bar{\alpha}_{n} \ldots \bar{\alpha}_{1} 0 \alpha_{1} \ldots \alpha_{n}$ where $\bar{\alpha}_{i}=-\alpha_{i}$. Then $D_{1}^{r}$ is just the usual Knuth (plactic) relation on the palindrome representation of $\alpha$ for any non negative integer $r$. On the other hand it is easy to see that when $r=1, D_{2}^{r}$ and $D_{3}^{r}$ are also usual Knuth relation on the palindrome representation of $\alpha$.

In this paper, the descriptions of Barbash-Vogan and Garfinkle's algorithms can be found in Section 2 together with some lemmas which are essential in the proofs of our results. Section 3 is devoted to the proof of Theorem 1.2

\section{Related background}

A sequence $\lambda=\left(\lambda_{1}, \ldots, \lambda_{k}\right)$ is a partition of $n$, denoted by $\lambda \vdash n$, if $\sum_{i=1}^{k} \lambda_{i}=n$ and $\lambda_{i} \geq \lambda_{i+1}>0$ where its Ferrers diagram consists of left justified arrows of boxes such that the $i$-th row has $\lambda_{i}$ boxes.

A partition $\lambda=\left(\lambda_{1}, \ldots, \lambda_{k}\right)$ can be also seen as a set of integer pairs $(i, j)$ such that $1 \leq i \leq k$ and $1 \leq j \leq \lambda_{i}$. Therefore for two partitions $\lambda$ and $\mu$, we can define usual set operations such as $\lambda \cup \mu, \lambda \cap \mu$, $\lambda \subset \mu, \lambda-\mu$, but the resulting sets do not necessarily correspond to some partitions.

Definition 2.1 For two partitions $\lambda$ and $\mu$ satisfying $\mu \subset \lambda$ we define $\lambda / \mu=\lambda-\mu$ to be the skew partition determined by $\lambda$ and $\mu$.

Definition 2.2 Let $\gamma$ and $\gamma^{\prime}$ be two skew shapes.

1. If $\gamma \cap \gamma^{\prime}=\emptyset$ and $\gamma \cup \gamma^{\prime}$ also corresponds a skew shape then we define $\gamma \oplus \gamma^{\prime}=\gamma \cup \gamma^{\prime}$.

2. If $\gamma^{\prime} \subset \gamma$ and $\gamma-\gamma^{\prime}$ also corresponds a skew shape then we define $\gamma \ominus \gamma^{\prime}=\gamma-\gamma^{\prime}$.

Definition 2.3 Let $\lambda$ be a partition and $(i, j) \in \lambda$.

1. If $(i, j) \in \lambda$ and $\lambda \ominus(i, j)$ is also a partition then $(i, j)$ is called a corner of $\lambda$.

2. If $(i, j) \notin \lambda$ and $\lambda \oplus(i, j)$ is also a partition then $(i, j)$ is called an empty corner of $\lambda$.

Definition 2.4 A skew tableau $T$ of shape $\lambda / \mu$ is obtained by labeling the cells of $\lambda / \mu$ with non repeating positive integers such that the numbers increase from left to right and from top to bottom. If $\mu=\emptyset$ then $T$ is called a Young tableau. We denote by label $(T)$ the set of numbers labeling each box of $T$ and by shape $(T)$ the partition underlying $T$. If the size of $\operatorname{shape}(T)=n$ and $\operatorname{label}(T)=\{1,2, \ldots, n\}$ then $T$ is called a standard skew or standard Young tableau according to the shape of $T$. We denote by $S Y T_{n}$ the set of all standard Young tableaux of $n$ cells. 
There is an important connection, between standard Young tableaux $S Y T_{n}$ and the symmetric group $S_{n}$, known as the Robinson-Schensted correspondence ( $R S K$ ), which was realized by Robinson and Schensted independently. In this correspondence, every permutation $w \in S_{n}$ is assigned bijectively to a pair of same shape tableaux $(P(w), Q(w))$ in $S Y T_{n} \times S Y T_{n}$ through insertion and recording algorithms. There are two equivalence relations introduced by Knuth which have very important applications in the combinatorics of tableaux.

Definition 2.5 For $u \in S_{n}$ consider the following relation: If $u_{i}<u_{i+2}<u_{i+1}$ or $u_{i}<u_{i-1}<u_{i+1}$ for some $i$ then $u=u_{1} \ldots u_{i-1}\left(u_{i} u_{i+1}\right) u_{i+2} \ldots u_{n} \stackrel{K}{\sim} u_{1} \ldots u_{i-1}\left(u_{i+1} u_{i}\right) u_{i+2} \ldots u_{n}=u^{\prime}$.

We say $u, w \in S_{n}$ are Knuth equivalent, $u \stackrel{K}{\sim} w$, if $w$ can be obtained from $u$ by applying a sequence of $\stackrel{K}{\sim}$ relations. On the other hand if $u^{-1} \stackrel{K}{\sim} w^{-1}$ then $u$ and $w$ are called dual Knuth equivalent, $u \stackrel{K^{*}}{\sim} w$.

The following theorem given by Knuth (12) is fundamental.

Theorem 2.6 Let $u, w \in S_{n}$. Then $u \stackrel{K}{\sim} w \Longleftrightarrow P(u)=P(w)$ and $u \stackrel{K^{*}}{\sim} w \Longleftrightarrow Q(u)=Q(w)$.

Definition 2.7 The set of two adjacent cells $A=\{(i, j),(i, j+1)\}$ (or $A=\{(i, j),(i+1, j)\})$ is called a horizontal (or respectively vertical) domino cell. By a labeling of domino cell $A$ we mean a pair of positive numbers $\left(a, a^{\prime}\right)$ which label the boxes of $A$ such that $a \leq a^{\prime}$ and a labels the cell of $A$ which is smaller in the lexicographic order. When we want to indicate the domino cell $A$ with its labeling, we use the notation

$$
\left[A,\left(a, a^{\prime}\right)\right]
$$

so that shape $\left(\left[A,\left(a, a^{\prime}\right)\right]\right)=A$ and $\operatorname{label}\left(\left[A,\left(a, a^{\prime}\right)\right]\right)=\left(a, a^{\prime}\right)$.

Let $\lambda$ be a partition and $A$ be a domino cell. If $\lambda \oplus A$ is a partition then $A$ is called an empty domino corner of $\lambda$ whereas if $\lambda \ominus A$ is also a partition then $A$ is called a domino corner of $\lambda$. Clearly, if a partition has no domino corner then it must be a $r$-staircase shape $(r, \ldots, 2,1)$ for some $r>0$. On the other hand it is easy to see that any partition $\lambda$ can be reduced uniquely to a $r$-staircase shape $(r, \ldots, 2,1)$ for some $r \geq 0$, by subsequent removal of existing domino corners one at a time. In this case we say $\lambda$ has a 2-core equivalent to $(r, \ldots, 2,1)$ and we denote by $P(2 n, r)$ the set of all such partitions of size $2 n+r(r+1) / 2$.

Definition 2.8 A $r$-domino tableau $T$ of shape $\lambda \in P(2 n, r)$ is obtained by tiling the skew partition $\lambda /(r, \ldots, 2,1)$ with labeled horizontal or vertical dominos $\left\{\left[A_{1},\left(a_{1}, a_{1}\right)\right], \ldots\left[A_{n},\left(a_{n}, a_{n}\right)\right]\right\}$ such that $a_{i} \neq a_{j}$ for $i \neq j$ and the labels increase from left to right and from top to bottom. In this case we have

$$
\operatorname{label}(T)=\left\{a_{1}, a_{2}, \ldots, a_{n}\right\} .
$$

A standard $r$-domino tableau $T$ is a $r$-domino tableau which has label $(T)=\{1, \ldots, n\}$. We denote by $S D T^{r}(n)$ the set of all standard $r$-domino tableaux of $n$ dominos.

Definition 2.9 Let $T$ be a $r$-domino tableau and $\lambda=\operatorname{shape}(T)$. For $a \in \operatorname{label}(T)$ and $A$ is a domino cell in $\lambda$ we define,

1. $\operatorname{Dom}(T, a)$ to be the domino cell of $T$ whose both cells are labeled with a in $T$.

2. $\operatorname{dom}(T, a)=\operatorname{shape}(\operatorname{Dom}(T, a))$.

3. label $(T, A)$ to be the pair of integers $\left(a, a^{\prime}\right)$ which label the domino cell $A$ in $T$, where $a \leq a^{\prime}$. 
Example 2.10 For example the following is a 2-domino tableau in $S D^{2}(5)$.

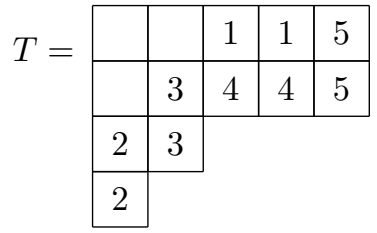

Thas two domino corners: $A_{1}=\{(1,5),(2,5)\}$ and $A_{2}=\{(2,4),(2,5)\}$. $\operatorname{label}\left(T, A_{1}\right)=(5,5)$ and $\operatorname{label}\left(T, A_{2}\right)=(4,5)$. On the other hand $\operatorname{dom}(T, 5)=A_{1}$ and $\operatorname{dom}(T, 4)=\{(2,3),(2,4)\} \neq A_{2}$.

Definition 2.11 For two $r$-domino tableau $S$ and $T$ satisfying $S \subset T$ we define $T / S=T-S$ to be the skew r-domino tableau determined by $S$ and $T$.

Definition 2.12 Let $R$ and $R^{\prime}$ be two skew $r$-domino tableaux with $\operatorname{shape}(R)=\gamma$ and $\operatorname{shape}\left(R^{\prime}\right)=\gamma^{\prime}$.

1. If $\gamma \oplus \gamma^{\prime}$ is defined and $R \cup R^{\prime}$ corresponds to some skew $r$-domino tableau as a set then we define $R \oplus R^{\prime}=R \cup R^{\prime}$

2. If $\gamma \ominus \gamma^{\prime}$ is defined and if $R-R^{\prime}$ corresponds to some skew $r$-domino tableau as a set then we define $R \ominus R^{\prime}=R-R^{\prime}$

Definition 2.13 Let $T$ be a (skew) $r$-domino tableau and $a \in \operatorname{label}(T)$. Then we define

1. $T_{<a}\left(T_{\leq a}\right)$ to be the $r$-domino tableau obtained by restricting $T$ to its dominos which are labeled with integers less than (and equal to) a.

2. $T_{>a}\left(T_{\geq a}\right)$ to be the skew $r$-domino tableau obtained by restricting $T$ to its dominos which are labeled with integers greater than (and equal to) a.

\subsection{Garfinkle's algorithm and reverse insertion}

Recall that a signed permutation $\alpha \in B_{n}$ is a bijection of $[-n,+n]$ such that $\alpha(-i)=-\alpha(i)$. The usual presentation of $\alpha \in B_{n}$ is denoted as $\alpha=\alpha_{1} \alpha_{2} \ldots \alpha_{n}$ where $\alpha_{i}=\alpha(i)$ for $1 \leq i \leq n$ and $\left\{\left|\alpha_{1}\right|,\left|\alpha_{2}\right| \ldots,\left|\alpha_{n}\right|\right\}=\{1,2, \ldots, n\}$.

In (4) Garfinkle provide an algorithm for $r=0,1$ by which any signed permutation $\alpha \in B_{n}$ is assigned bijectively to a pair of same shape standard $r$-domino tableau $\left(P^{r}(\alpha), Q^{r}(\alpha)\right)$, where $P^{r}(\alpha)$ is called insertion and $Q^{r}(\alpha)$ is called recording tableau of $\alpha$. Detailed explanations of this algorithm can be found in (13) and (21). Based on Garfinkle's algorithm we now describe the reverse-insertion of domino corners through the Corollary below. Then we will state several lemmas which are the main tool in the proof of Theorem 1.2

Let $T$ be a $r$-domino tableau and $A$ be a domino corner in shape $(T)$. We denote by $T^{\uparrow A}$ and $\eta\left(T^{\uparrow A}\right)$ respectively the tableau which is obtained by the reverse-insertion of $A$, and the number which is bumped out of $T$ as a result of this operation.

Corollary 2.14 Let $T$ be an $r$-domino tableau and $A$ is a domino corner. Furthermore let $A^{\prime}$ be the domino cell which is pushed back by $A$ in reverse insertion $T^{\uparrow A}$. Then, 
i) If $A=\{(i, j),(i, j+1)\}$ and label $(T, A)=(a, a)$ then $A^{\prime} \subset\{(i-1, k) \mid k \geq j\}$.

ii) If $A=\{(i, j),(i, j+1)\}$ and $\operatorname{label}(T, A)=\left(a^{\prime}, a\right)$ for some $a^{\prime}<a$ then $A^{\prime}=\{(i-1, j),(i, j)\}$.

iii) If $A=\{(i, j),(i+1, j)\}$ and $\operatorname{label}(T, A)=(a, a)$ then $A^{\prime} \subset\{(k, j-1) \mid k \geq i\}$.

iv) If $A=\{(i, j),(i+1, j)\}$ and $\operatorname{label}(T, A)=\left(a^{\prime}, a\right)$ for some $a^{\prime}<a$ then $A^{\prime}=\{(i, j-1),(i, j)\}$.

Example 2.15 Let $S \in S D^{3}(5)$ as given below. We will show that $\eta\left(S^{\uparrow A}\right)=1$ where $A=\{(3,3),(4,3)\}$. In the following the barred letters indicate the domino cell which is pushed back during the reverse insertion algorithm.

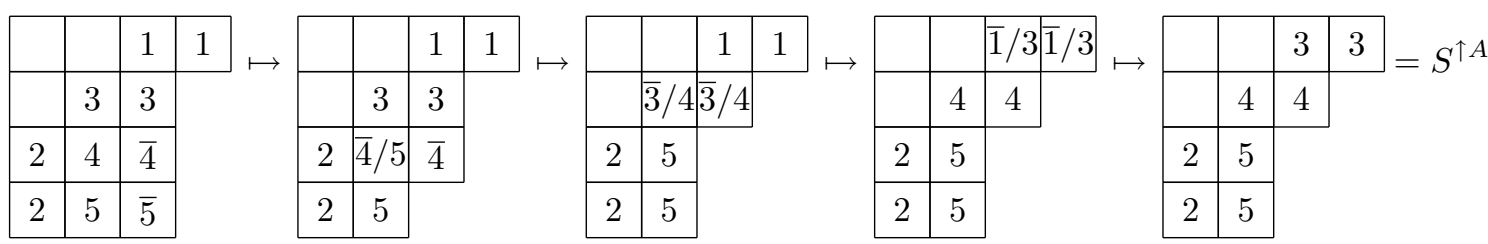

Lemma 2.16 Let $T$ be a $r$-domino tableau and $A$ be a domino corner of $\operatorname{shape}(T)$. Then $T^{\uparrow A}$ and $\eta\left(T^{\uparrow A}\right)$ are unique.

Definition 2.17 Let $T$ be a $r$-domino tableau and $A$ be a domino corner of shape $(T)$ such that $A=$ $\{(i, j),(i, j+1)\}$ or $A=\{(i, j),(i+1, j)\}$. We denote by $(T, A$, ne $)$ and $(T, A$, ne) the regions of $T$ such that

$$
\begin{aligned}
& (T, A, \text { ne }) \quad:=\{(k, l) \mid k<i \text { and } l \geq j\} \\
& (T, A, \mathrm{sw}) \quad:=\{(k, l) \mid k \geq i \text { and } l<j\}
\end{aligned}
$$

as illustrated in Figure 1

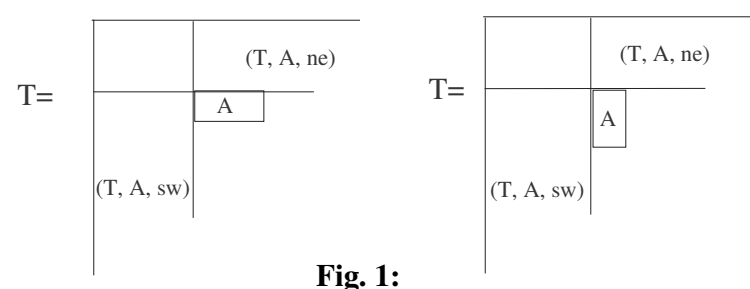

Fig. 1:

Lemma 2.18 Let $A$ and $B$ be a domino corners of shape $(T)$ and shape $\left(T^{\uparrow A}\right)$ respectively.

i) If $B$ lies in the portion $(T, A$, sw $)$ then $\eta\left(T^{\uparrow A \uparrow B}\right)<\eta\left(T^{\uparrow A}\right)$.

ii) If $B$ lies in the portion $\left(T, A\right.$, ne) then $\eta\left(T^{\uparrow A \uparrow B}\right)>\eta\left(T^{\uparrow A}\right)$.

Proof: The proof is omitted for the sake of place. 


\subsection{Barbash and Vogan algorithm and descents of $r$-domino tableaux.}

We will now explain the algorithm which is provided by Barbash and Vogan in (1) to establish the bijection between signed permutations and standard $r$-domino tableaux for $r=0,1$ whereas its extension for larger cores is provided in (3). We also remark that the equivalence of Barbash-Vogan algorithm to Garfinkle's algorithm for $r=0,1$, is due to Van Leeuwen (14).

Recall that for $\alpha=\alpha_{1} \alpha_{2} \ldots \alpha_{n}$ a signed permutation the palindrome representation of $\alpha$ is given by $\alpha^{0}=\bar{\alpha}_{n} \ldots \bar{\alpha}_{2} \bar{\alpha}_{1} \alpha_{1} \alpha_{2} \ldots \alpha_{n}$ if $\alpha$ lies in $C_{n}$, or $\alpha^{1}=\bar{\alpha}_{n} \ldots \bar{\alpha}_{2} \bar{\alpha}_{1} 0 \alpha_{1} \alpha_{2} \ldots \alpha_{n}$ if $\alpha$ lies in $B_{n}$, where $\bar{\alpha}_{i}=-\alpha_{i}$. We will call $\alpha^{0}$ and $\alpha^{1}$ as 0 -core and 1-core representation of $\alpha$ respectively. By following the approach of (3) let us describe how to extend this representation for larger cores. We first identify $\{1,2, \ldots, r(r+1) / 2\}$ with $\left\{0_{1}, 0_{2}, \ldots, 0_{r(r+1) / 2}\right\}$ together with the total ordering $-n<\ldots<$ $-2<-1<0_{1}<0_{2}<\ldots<0_{r(r+1) / 2}<1<2 \ldots<n$. Let $w \in S_{r(r+1) / 2}$ be a permutation under this identification, whose RSK insertion tableau is of shape $(r, r-1, \ldots, 1)$. Now for $\alpha \in B_{n}$ let $r$-core representation of $\alpha$ to be $\alpha^{r}=\bar{\alpha}_{n} \ldots \bar{\alpha}_{2} \bar{\alpha}_{1} w \alpha_{1} \alpha_{2} \ldots \alpha_{n}$. The algorithm first applies RSK algorithm on $\alpha^{r}$. Then starting from the lowest number $\bar{n}$, it vacates the negative integer $\bar{i}$ in the tableaux by jeu de taquin slides until it becomes adjacent to $i$, where the vacation is repeated for $\overline{i-1}$ until $i=1$. The following example illustrates this algorithm for $r=1$.

Example 2.19 For $\alpha=3 \overline{1} 2 \in B_{n}$, we have $\alpha^{1}=\overline{2} 1 \overline{3} 03 \overline{1} 2$ be its 1 -core representation. Then

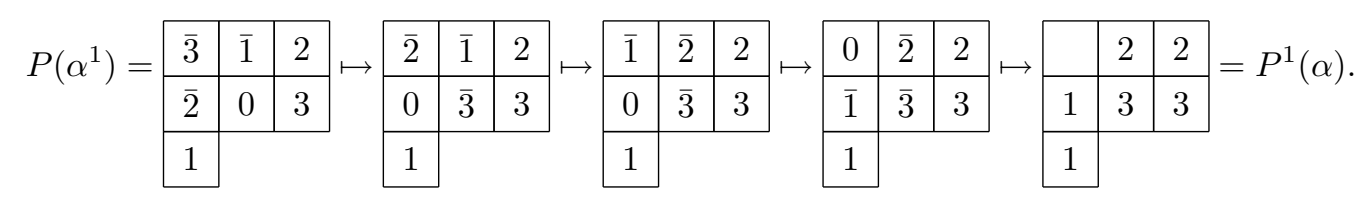

Theorem 2.20 ([3), Theorem 3.3) Signed permutations $\alpha$ and $\beta$ have the same insertion $r$-domino tableau if and only if $\alpha^{r}$ and $\beta^{r}$ have the same RSK insertion tableau.

The following proposition is a consequence of Theorem 2.20 and Theorem 2.6

Proposition 2.21 Let $\alpha$ and $\beta$ be two signed permutations which differ by a single $D_{1}^{r}$ relation. Then $P^{r}(\alpha)=P^{r}(\beta)$, in other words $\alpha$ and $\beta$ have the same insertion $r$-domino tableau.

Recall that $B_{n}$ carries a Coxeter group structure with the generator set $S=\left\{s_{0}, s_{1}, \ldots, s_{n-1}\right\}$ where $\left\{s_{i}=(i, i+1) \mid 1 \leq i \leq n-1\right\}$ is the set of transpositions which also generates the symmetric group $S_{n}$ and $s_{0}$ corresponds to the transposition $(-1,1)$. Let $l(\alpha)$ denote the length of $\alpha$, which is the minimum number of generators of $\alpha$ and let

$$
\begin{aligned}
\operatorname{Des}_{L}(\alpha) & :=\left\{i \mid l\left(s_{i} \alpha\right)<l(\alpha) \text { and } 0 \leq i \leq n-1\right\} \\
& =\left\{i \mid \text { if } 1 \leq i \leq n-1 \text { and } i+1 \text { comes before } i \text { in } \alpha^{0}\right\} \cup\left\{0 \mid \text { if } 1 \text { comes before }-1 \text { in } \alpha^{0}\right\}
\end{aligned}
$$

denote respectively the sets of left and right descents of $\alpha$. On the other hand the descent set of a $r$-domino tableau $T$ is defined in the following way:

$$
\begin{aligned}
\operatorname{Des}(T):= & \{i \mid \text { if the domino labeled with }(i+1, i+1) \text { lies below the one labeled with }(i, i)\} \\
& \cup\{0 \mid \text { if the domino labeled with }(1,1) \text { is vertical }\}
\end{aligned}
$$

It is a well known property of RSK algorithm that $\operatorname{Des}_{L}(w)=\operatorname{Des}(P(w))$ for any $w \in S_{n}$ whereas the descent set of a (skew or Young) tableau $T$ is defined by $\operatorname{Des}(T)=\{i \mid i+1$ lies below $i$ in $T\}$. On the 
other hand it is easy to see that jeu de taquin slides do not change the descent sets of tableaux, therefore the following result is a consequence of Theorem 2.20 .

Corollary 2.22 For $\alpha \in B_{n}$ we have $\operatorname{Des}_{L}(\alpha)=\operatorname{Des}\left(P^{r}(\alpha)\right)$.

Observe that if $\alpha$ and $\beta$ differ by a single $D_{1}^{r}$ relations in $B_{n}$ then $P^{r}(\alpha)=P^{r}(\beta)$ and we have either $\beta^{-1}=s_{i} \cdot \alpha^{-1}$ or $\beta^{-1}=s_{i+1} \cdot \alpha^{-1}$ and moreover we have either $i \in \operatorname{Des}_{L}\left(\alpha^{-1}\right)$ but $i+1 \notin \operatorname{Des}_{L}\left(\alpha^{-1}\right)$ or $i \notin \operatorname{Des}_{L}\left(\alpha^{-1}\right)$ but $i+1 \in \operatorname{Des}_{L}\left(\alpha^{-1}\right)$ for some $1 \leq i \leq n-2$. In the following we will follow Garfinkle's approach in (4) to study the effect of a single $D_{1}^{r}$ relation on the recording tableaux.

For $i, j$ two adjacent integers satisfying $1 \leq i, j \leq n-1$, consider the following sets:

$$
\begin{gathered}
D_{i, j}\left(B_{n}\right):=\left\{\alpha \in B_{n} \mid i \in \operatorname{Des}_{L}(\alpha) \text { but } j \notin \operatorname{Des}_{L}(\alpha)\right\} \\
D_{i, j}\left(S D T^{r}(n)\right):=\left\{T \in S D T^{r}(n) \mid i \in \operatorname{Des}(T) \text { but } j \notin \operatorname{Des}(T)\right\}
\end{gathered}
$$

together with the map $V_{i, j}: D_{i, j}\left(B_{n}\right) \mapsto D_{j, i}\left(B_{n}\right)$ where $V_{i, j}(\alpha)=\left\{s_{i} \cdot \alpha, s_{j} \cdot \alpha\right\} \cap D_{j, i}\left(B_{n}\right)$. We also define a map $V_{i, j}: D_{i, j}\left(S D T^{r}(n)\right) \mapsto D_{j, i}\left(S D T^{r}(n)\right)$ in the following manner: Without loss of generality we assume that $j>i$, i.e., $j=i+1$. Observe that if $i \in \operatorname{Des}(T)$ but $i+1 \notin \operatorname{Des}(T)$ then $i+1$ lies strictly below $i$ in $T$ whereas $i+2$ lies strictly right to $i+1$ in $T$. On the other hand we have two cases according to the positions of dominos labeled with $(i, i)$ and $(i+2, i+2)$ with respect to each other.

Case 1. We first assume that $i+2$ lies strictly below $i$ in $T$. Since the $i+2$ lies strictly to the right of $i+1$ and $i+1$ lies below $i$ we have two cases to consider: If the boundaries $\operatorname{Dom}(T, i+1)$ and $\operatorname{Dom}(T, i)$ intersect at most on a point then $V_{i, i+1}(T)$ is obtained by interchanging the labels $i$ and $i+1$ in $T$. Otherwise there is only one possibility which satisfies $i+2$ lies below $i$ and it lies to the right of $i+1$, in which $T$ has the subtableau $U$ as illustrated below and $V_{i, i+1}(T)$ is obtained by substituting $U$ with $U^{\prime}$ in $T$.

$$
U=\begin{array}{|c|c|}
\hline i & i \\
\hline i+1 & i+2 \\
\hline i+1 & i+2 \\
\hline
\end{array} \quad U^{\prime}=\begin{array}{|c|c|}
\hline i & i+1 \\
\hline i & i+1 \\
\hline i+2 & i+2 \\
\hline
\end{array}
$$

Case 2. Now we assume $i+2$ lies strictly right to $i$ in $T$. Again if the boundaries of $\operatorname{Dom}(T, i+1)$ and $\operatorname{Dom}(T, i+2)$ intersect at most on a point then $V_{i, i+1}(T)$ is obtained by interchanging the labels $i+1$ and $i+2$ in $T$. Otherwise there is only one possible case where $T$ has the subtableau $U$ given below and $V_{i, i+1}(T)$ is obtained by substituting $U$ with $U^{\prime}$ in $T$.

$$
U=\begin{array}{|c|c|c|}
\hline i & i & i+2 \\
\hline i+1 & i+1 & i+2 \\
\hline
\end{array} \quad U^{\prime}=\begin{array}{|c|c|c|}
\hline i & i+1 & i+1 \\
\hline i & i+2 & i+2 \\
\hline
\end{array}
$$

Example 2.23 We have $T_{2}=V_{5,6}\left(T_{1}\right), T_{3}=V_{3,4}\left(T_{2}\right)$, and $T_{4}=V_{4,5}\left(T_{3}\right)=V_{6,5}\left(T_{3}\right)$ for the following tableaux.

$$
T_{1}=\begin{array}{|l|l|l|}
\hline 1 & 2 & \mathbf{5} \\
\hline 1 & 2 & \mathbf{5} \\
\hline 3 & 3 & \mathbf{7} \\
\hline 4 & \mathbf{6} & \mathbf{7} \\
\hline 4 & \mathbf{6}
\end{array} \quad T_{2}=\begin{array}{|l|l|l|}
\hline 1 & 2 & 6 \\
\hline 1 & 2 & 6 \\
\hline \mathbf{3} & \mathbf{3} & 7 \\
\hline \mathbf{4} & \mathbf{5} & 7 \\
\hline \mathbf{4} & \mathbf{5} \\
\hline
\end{array} \quad T_{3}=\begin{array}{|l|l|l|}
\hline 1 & 2 & \mathbf{6} \\
\hline 1 & 2 & \mathbf{6} \\
\hline 3 & \mathbf{4} & \mathbf{7} \\
\hline 3 & \mathbf{4} & \mathbf{7} \\
\hline \mathbf{5} & \mathbf{5} \\
\end{array} \quad T_{4}=\begin{array}{|l|l|l|l|}
\hline 1 & 2 & 5 \\
\hline 1 & 2 & 5 \\
\hline 3 & 4 & 7 \\
\hline 3 & 4 & 7 \\
\hline 6 & 6 &
\end{array}
$$


Remark 2.24 The map $V_{i, j}$ is first introduced on the symmetric group by Vogan (22), with the aim of classifying the primitive ideals in the universal enveloping algebra of complex semi simple Lie algebras. In fact when it is considered on the symmetric group the map $V_{i, j}$ produces nothing but the dual Knuth relation on the permutations and their insertion tableaux.

Lemma 2.25 Let $i$ and $j$ be two consecutive integers such that $1 \leq i, j \leq n-1$. Suppose $\alpha \in D_{i, j}\left(B_{n}\right)$. Then $P^{r}(\alpha) \in D_{i, j}\left(S D T^{r}(n)\right)$ and $P^{r}\left(V_{i, j}(\alpha)\right)=V_{i, j}\left(P^{r}(\alpha)\right)$.

Proof: This result is first proven by Garfinkle (5, Theorem 2.1.19.) for $r=0,1$. On the other hand one can check that her proof does not depend on the specific value of $r$ and it can easily be extended for any value of $r$. We omit the proof for the sake of space.

The following result follows directly from Lemma 2.25 and it has an important role in the proof of Theorem 1.2

Corollary 2.26 Suppose $\alpha=\alpha_{1} \ldots \alpha_{i-1}\left(\alpha_{i} \alpha_{i+1}\right) \alpha_{i+2} \ldots \alpha_{n}$ and $\beta=\alpha_{1} \ldots \alpha_{i-1}\left(\alpha_{i+1} \alpha_{i}\right) \alpha_{i+2} \ldots \alpha_{n}$ differ by a single $\mathrm{D}_{1}^{r}$ relation. Then one of the following is satisfied:

i) $\alpha_{i}<\alpha_{i+2}<\alpha_{i+1}$ for some $i \leq n-2$ and $\beta^{-1}=V_{i+1, i}\left(\alpha^{-1}\right)$ and $Q^{r}(\beta)=V_{i+1, i}\left(Q^{r}(\alpha)\right)$.

ii) $\alpha_{i}>\alpha_{i+2}>\alpha_{i+1}$ for some $i \leq n-2$ and $\beta^{-1}=V_{i, i+1}\left(\alpha^{-1}\right)$ and $Q^{r}(\beta)=V_{i, i+1}\left(Q^{r}(\alpha)\right)$.

iii) $\alpha_{i}<\alpha_{i-1}<\alpha_{i+1}$ for some $i \leq n-1$ and $\beta^{-1}=V_{i-1, i}\left(\alpha^{-1}\right)$ and $Q^{r}(\beta)=V_{i-1, i}\left(Q^{r}(\alpha)\right)$.

iv) $\alpha_{i}>\alpha_{i-1}>\alpha_{i+1}$ for some $i \leq n-1$ and $\beta^{-1}=V_{i, i-1}\left(\alpha^{-1}\right)$ and $Q^{r}(\beta)=V_{i, i-1}\left(Q^{r}(\alpha)\right)$.

\section{Plactic relations for $r$-domino tableaux}

\subsection{Proof of Theorem 1.2}

In this section we will prove the main Theorem 1.2 , i.e., we will show that the relations $\mathrm{D}_{1}^{r}, \mathrm{D}_{2}^{r}$ and $\mathrm{D}_{3}^{r}$ from Definition 1.1 are sufficient and necessary to characterize plactic classes of standard $r$-domino tableaux.

Proof: ( Proof of Theorem 1.2) Let $\alpha$ and $\beta$ be two signed permutations which differ by a sequence of $\mathrm{D}_{1}^{r}$, $\mathrm{D}_{2}^{r}$ or $\mathrm{D}_{3}^{r}$ relations. By using Garfinkle's insertion algorithm for $r$-domino tableau and Proposition 2.21 it is easy to check that $P^{r}(\alpha)=P^{r}\left(\alpha^{\prime}\right)$ if $\alpha$ and $\alpha^{\prime}$ differs by a single $\mathrm{D}_{i}^{r}$ relation for $i=1,2,3$. Therefore $P^{r}(\alpha)$ must be equal to $P^{r}(\beta)$.

Now we let $\alpha=\alpha_{1} \ldots \alpha_{n-1} \alpha_{n}$ and $\beta=\beta_{1} \ldots \beta_{n-1} \beta_{n}$ such that $T=P^{r}(\alpha)=P^{r}(\beta)$. We will show by induction that $\alpha \stackrel{p_{r}}{\sim} \beta$. Let $r \geq 0$ be arbitrary. If $n=1$ there is nothing to prove. Therefore we assume that the statement holds for all signed permutations of size $n-1$.

If $P^{r}\left(\alpha_{1} \ldots \alpha_{n-1}\right)=P^{r}\left(\beta_{1} \ldots \beta_{n-1}\right)=T^{\uparrow A}$ for some domino corner $A$ of shape $(T)$ then $\alpha_{n}=\beta_{n}$ by Lemma 2.16 By induction we can assume that $\alpha_{1} \ldots \alpha_{n-1} \stackrel{p_{r}}{\sim} \beta_{1} \ldots \beta_{n-1}$. Therefore $\alpha \stackrel{p_{r}}{\sim} \beta$.

If $P^{r}\left(\alpha_{1} \ldots \alpha_{n-1}\right) \neq P^{r}\left(\beta_{1} \ldots \beta_{n-1}\right)$ then there exist two different domino corners say $A$ and $B$ of $T$ such that

$$
\begin{aligned}
& T^{\uparrow A}=P^{r}\left(\alpha_{1} \ldots \alpha_{n-1}\right) \text { and } \eta\left(T^{\uparrow A}\right)=\alpha_{n} \\
& T^{\uparrow B}=P^{r}\left(\beta_{1} \ldots \beta_{n-1}\right) \text { and } \eta\left(T^{\uparrow B}\right)=\beta_{n} .
\end{aligned}
$$


In the following we proceed according to the orientation of $A$ and $B$ as illustrated in Figure 2 where in the first four pictures $(T, A, \mathrm{ne}) \cap(T, B, \mathrm{sw})$ is represented with the shaded areas.

(1)

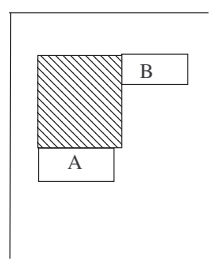

(2)

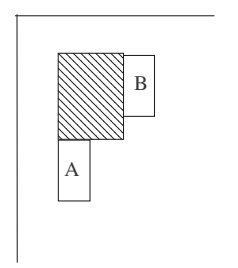

(3)

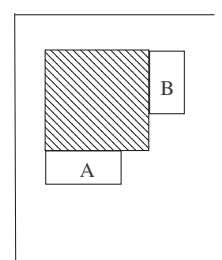

(4)

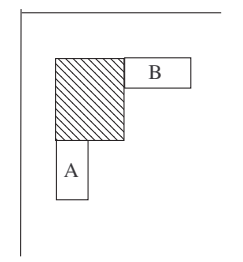

(5)

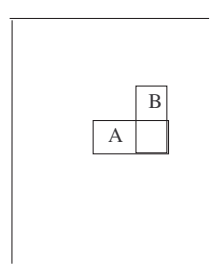

Fig. 2:

Cases (1),(2) and (3): We will first show that $\alpha \stackrel{p_{r}}{\sim} \beta$ for the first three cases of Figure 2 Consider the domino corner $B$ of $T^{\uparrow A}$ and let $b=\eta\left(T^{\uparrow A \uparrow B}\right)$. It is easy to see that there exists a domino corner, say $C$ of $T^{\uparrow A \uparrow B}$ which lies in $(T, A, \mathrm{ne}) \cap(T, B, \mathrm{sw})$. Let $c=\eta\left(T^{\uparrow A \uparrow B \uparrow C}\right)$ and $\tilde{u}$ be a signed word such that $P^{r}(\tilde{u})=T^{\uparrow A \uparrow B \uparrow C}$. Therefore by Lemma 2.16 we have

$$
P^{r}\left(\tilde{u} c b \alpha_{n}\right)=P^{r}(\tilde{u})^{\downarrow c \downarrow b \downarrow \alpha_{n}}=\left(T^{\uparrow A \uparrow B \uparrow C}\right)^{\downarrow c \downarrow b \downarrow \alpha_{n}}=\left(T^{\uparrow A \uparrow B}\right)^{\downarrow b \downarrow \alpha_{n}}=\left(T^{\uparrow A}\right)^{\downarrow \alpha_{n}}=T
$$

and by induction hypothesis $\tilde{u} c b \stackrel{p_{r}}{\sim} \alpha_{1} \ldots \alpha_{n-1}$ since $P^{r}(\tilde{u} c b)=T^{\uparrow A}=P^{r}\left(\alpha_{1} \ldots \alpha_{n-1}\right)$. Therefore letting $u$ denote the signed permutation $\tilde{u} c b \alpha_{n}$, we have $\alpha \stackrel{p_{r}}{\sim} u$.

Observe that since $P^{r}(\tilde{u})=T^{\uparrow A \uparrow B \uparrow C}$, the recording tableau $Q^{r}\left(\tilde{u} c b \alpha_{n}\right)$ has its domino cells $A, B$ and $C$ labeled with $(n, n),(n-1, n-1)$ and $(n-2, n-2)$ respectively.

On the other hand having $B$ in $(T, A$, ne) and $C$ in $(T, B, \mathrm{sw})$ yields by Lemma 2.18 that

$$
b=\eta\left(T^{\uparrow A \uparrow B}\right)>\eta\left(T^{\uparrow A}\right)=\alpha_{n} \text { and } b=\eta\left(T^{\uparrow A \uparrow B}\right)>\eta\left(T^{\uparrow A \uparrow B \uparrow C}\right)=c .
$$

Therefore we have by Corallary 2.26

$$
\begin{aligned}
& \text { either } \quad b>\alpha_{n}>c, \text { and hence } u=\tilde{u} c b \alpha_{n} \stackrel{\mathrm{D}_{1}^{r}}{\sim} \tilde{u} b c \alpha_{n}=w \text { and } V_{n-1, n-2}\left(Q^{r}(u)\right)=Q^{r}(w) \\
& \text { or } \quad b>c>\alpha_{n}, \text { and hence } u=\tilde{u} c b \alpha_{n} \stackrel{\mathrm{D}_{1}^{r}}{\sim} \tilde{u} c \alpha_{n} b=w \text { and } V_{n-1, n-2}\left(Q^{r}(u)\right)=Q^{r}(w)
\end{aligned}
$$

The last argument implies that in both cases the signed permutation $w$ has its recording tableau $Q^{r}(w)$ obtained by interchanging the labels $(n, n)$ of $A$ and $(n-1, n-1)$ of $B$ in $Q^{r}(u)$ i.e., $Q^{r}(w)$ had the domino corner $B$ labeled with $(n, n)$. Then by Lemma 2.16 we have

$$
P^{r}\left(w_{1} \ldots w_{n-1}\right)=T^{\uparrow B}=P^{r}\left(\beta_{1} \ldots \beta_{n-1}\right) \text { and } w_{n}=\beta_{n}
$$

and by induction $w_{1} \ldots w_{n-1} \stackrel{p_{r}}{\sim} \beta_{1} \ldots \beta_{n-1}$. Therefore $w \stackrel{p_{r}}{\sim} \beta$. and $\alpha \stackrel{p_{r}}{\sim} u \stackrel{p_{r}}{\sim} w \stackrel{p_{r}}{\sim} \beta$.

Case (4): For the fourth case of Figure 2, let $\alpha, \beta \in B_{n}$ as in 1. If there exist a domino corner in $(T, A, \mathrm{ne}) \cap(T, B, \mathrm{sw})$ then one can follow the same argument which is used for Cases (1),(2),(3). On the other hand it may happen that $(T, A, \mathrm{ne}) \cap(T, B, \mathrm{sw})$ is a staircase shape and in the following we consider several subcases as illustrated in Figure 3 
(a)

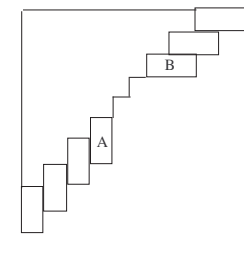

(b)

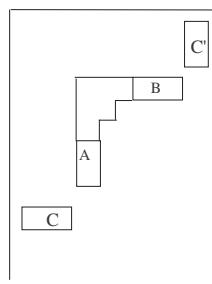

(c)

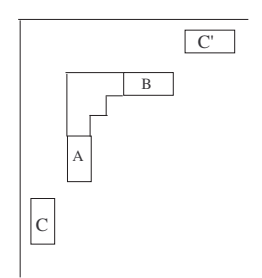

(d)

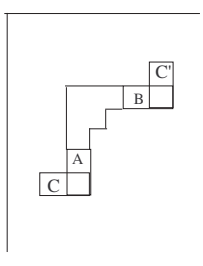

Fig. 3:

Observe that, in case $T$ has the configuration of Figure 3 a), we have $n \leq r+1, \alpha_{n}<0, \beta_{n}>0$ and

$$
\begin{aligned}
\eta\left(T^{\uparrow A \uparrow B}\right) & =\beta_{n} \\
\eta\left(T^{\uparrow B \uparrow A}\right) & =\alpha_{n} \\
P^{r}\left(\alpha_{1} \ldots \alpha_{n-2}\right)=T^{\uparrow \uparrow \uparrow B} & =T^{\uparrow \uparrow \uparrow A}=P^{r}\left(\beta_{1} \ldots \beta_{n-2}\right) .
\end{aligned}
$$

Let $\tilde{u}$ be a signed word such that $P^{r}(\tilde{u})=T^{\uparrow A \uparrow B}=T^{\uparrow B \uparrow A}$. Clearly $\tilde{u}$ must be a shuffle of positive decreasing and negative increasing sequences and $P^{r}\left(\tilde{u} \alpha_{n} \beta_{n}\right)=T=P^{r}\left(\tilde{u} \beta_{n} \alpha_{n}\right)$. Therefore $\tilde{u} \alpha_{n} \beta_{n} \stackrel{\mathrm{D}_{2}^{r}}{\sim} \tilde{u} \beta_{n} \alpha_{n}$. On the other hand $P^{r}\left(\tilde{u} \beta_{n}\right)=T^{\uparrow A}$ and $P^{r}\left(\tilde{u} \alpha_{n}\right)=T^{\uparrow B}$ and by induction hypothesis we have $\alpha_{1} \ldots \alpha_{n-1} \stackrel{p_{r}}{\sim} \tilde{u} \beta_{n}$ and $\beta_{1} \ldots \beta_{n-1} \stackrel{p_{r}}{\sim} \tilde{u} \alpha_{n}$. Hence

$$
\alpha=\alpha_{1} \ldots \alpha_{n-1} \alpha_{n} \stackrel{p_{r}}{\sim} \tilde{u} \beta_{n} \alpha_{n} \stackrel{\mathrm{D}_{2}^{r}}{\sim} \tilde{u} \alpha_{n} \beta_{n} \stackrel{p_{r}}{\sim} \beta_{1} \ldots \beta_{n-1} \beta_{n}=\beta .
$$

Now we assume that $T$ has the configuration of Figure 3 d) i.e. the corner $C$ and $A$ (or $C^{\prime}$ and $B$ ) intersect. Again we let $\sigma_{1} \ldots \sigma_{n} \in B_{n}$ such that $P^{r}\left(\sigma_{1} \ldots \sigma_{n-1}\right)=T^{\uparrow C}$. Observe that there is a domino corner in $(T, C, \mathrm{ne}) \cap(T, B, \mathrm{sw})$ therefore $\beta \stackrel{p_{r}}{\sim} \sigma$ follows. We only need to show $\alpha \stackrel{p_{r}}{\sim} \sigma$.

Observe that since $T$ has the configuration of Figure 3(d) we have a domino corner $A^{\prime}$ of $T^{\uparrow A}$ and $A^{\prime \prime}$ of $T^{\uparrow A \uparrow A^{\prime}}$ as it is illustrated in Figure 4 below.
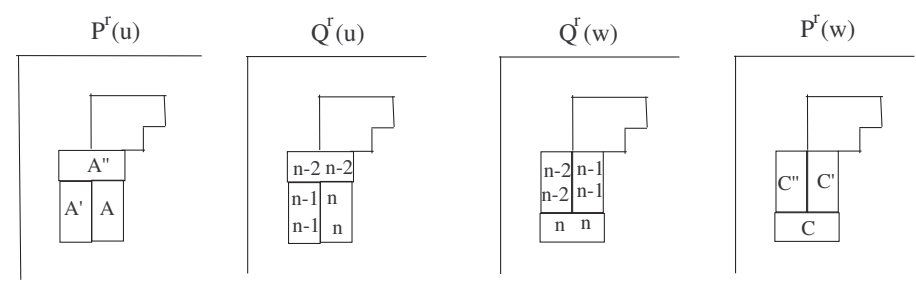

Fig. 4:

Let $a^{\prime}=\eta\left(T^{\uparrow A \uparrow A^{\prime}}\right)$ and $a^{\prime \prime}=\eta\left(T^{\uparrow A \uparrow A^{\prime} \uparrow A^{\prime \prime}}\right)$. Suppose $\tilde{u}$ be a signed word such that $P^{r}(\tilde{u})=$ $T^{\uparrow A \uparrow A^{\prime} \uparrow A^{\prime \prime}}$. Then the signed permutation $u=\tilde{u} a^{\prime \prime} a^{\prime} \alpha_{n}$ has $P^{r}(u)=T$ whereas its recording tableau $Q^{r}(u)$ must have the form as it is shown in Figure 4.

On the other hand since $P^{r}\left(\tilde{u} a^{\prime \prime} a\right)=T^{\uparrow A}=P^{r}\left(\alpha_{1} \ldots \alpha_{n-1}\right)$ we have by induction $\tilde{u} a^{\prime \prime} a^{\prime} \stackrel{p_{r}}{\sim} \alpha_{1} \ldots \alpha_{n-1}$ and therefore $u=\tilde{u} a^{\prime \prime} a^{\prime} \alpha_{n} \stackrel{p_{r}}{\sim} \alpha_{1} \ldots \alpha_{n-1} \alpha_{n}=\alpha$. 
Furthermore having $A^{\prime}$ in $(T, A$, sw $)$ and $A^{\prime \prime}$ in $\left(T, A^{\prime}\right.$, ne) yields $a^{\prime}=\eta\left(T^{\uparrow A \uparrow A^{\prime}}\right)<\eta\left(T^{\uparrow A}\right)=$ $\alpha_{n}$ and $a^{\prime}=\eta\left(T^{\uparrow A \uparrow A^{\prime}}\right)<\eta\left(T^{\uparrow A \uparrow A^{\prime} \uparrow A^{\prime \prime}}\right)=a^{\prime \prime}$ by Lemma 2.18. Therefore we have

either $\quad a^{\prime \prime}>\alpha_{n}>a^{\prime}$, and hence $u=\tilde{u} a^{\prime \prime} a^{\prime} \alpha_{n} \stackrel{\mathrm{D}_{1}^{r}}{\sim} \tilde{u} a^{\prime} a^{\prime \prime} \alpha_{n}=w$ and $Q^{r}(w)=V_{n-2, n-1}\left(Q^{r}(u)\right)$

or $\quad \alpha_{n}>a^{\prime \prime}>a^{\prime}$, and hence $u=\tilde{u} a^{\prime \prime} a^{\prime} \alpha_{n} \stackrel{\mathrm{D}_{1}^{r}}{\sim} \tilde{u} a^{\prime \prime} \alpha_{n} a^{\prime}=w$ and $Q^{r}(w)=V_{n-2, n-1}\left(Q^{r}(u)\right)$.

In both cases Corollary 2.26 yields that the recording tableau $Q^{r}(w)$ of $w$ has the form illustrated in Figure 4 and by Lemma 2.16 we have

$$
P^{r}\left(w_{1} \ldots w_{n-1}\right)=T^{\uparrow C}=P^{r}\left(\sigma_{1} \ldots \sigma_{n-1}\right) \text { and } w_{n}=\beta_{n} .
$$

Then by induction $w_{1} \ldots w_{n-1} \stackrel{p_{r}}{\sim} \sigma_{1} \ldots \sigma_{n-1}$ and hence $w \stackrel{p_{r}}{\sim} \sigma$. Hence as desired $\alpha \stackrel{p_{r}}{\sim} u \stackrel{\mathrm{D}_{1}^{r}}{\sim} w \stackrel{p_{r}}{\sim} \beta$.

If $T$ has the configuration of Figure 3 (b) or Figure $3(\mathrm{c}), T$ may have a domino corner, say $C$ lying in $\left(T, A\right.$, ne). Let $\sigma=\sigma_{1} \ldots \sigma_{n} \in B_{n}$ such that $=P^{r}\left(\sigma_{1} \ldots \sigma_{n-1}\right)=T^{\uparrow C}$. Observe that there exist a domino corner in $(T, C, \mathrm{ne}) \cap(T, A, \mathrm{sw})$ and $(T, C, \mathrm{ne}) \cap(T, B, \mathrm{sw})$ therefore we can apply the argument which is used for Cases (1),(2) and (3) in order to get $\alpha \stackrel{p_{r}}{\sim} \sigma$ and $\beta \stackrel{p_{r}}{\sim} \sigma$ and hence $\alpha \stackrel{p_{r}}{\sim} \beta$. On the other hand if $T$ has domino corner $C^{\prime}$ lying in $(T, B, \mathrm{sw})$ the same argument applied on $T^{t}$ gives the desired result.

Case (5): Again let $\alpha, \beta \in B_{n}$ as in 1 and suppose that $T$ has the configuration of Figure 2 (5) . We consider Figure 5 for several cases.

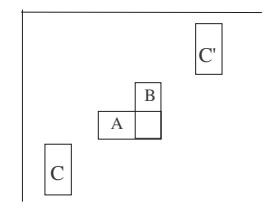

(a)
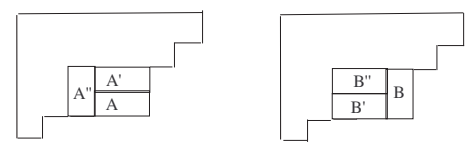

(b)
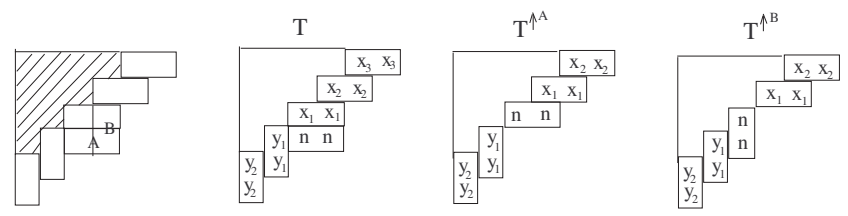

(c)

Fig. 5: 
If $T$ has a corner, say $C$, lying in $\left(T, A\right.$, sw), as it is illustrated in Figure 5 a), let $\sigma_{1} \ldots \sigma_{n} \in B_{n}$ such that

$$
P^{r}\left(\sigma_{1} \ldots \sigma_{n-1}\right)=T^{\uparrow C} .
$$

Since there is a domino corner in $(T, C, \mathrm{ne}) \cap(T, B, \mathrm{sw})$ we have $\beta \stackrel{p_{r}}{\sim} \sigma$ as in the Cases (1),(2) and (3). If $C$ is a vertical domino corner, the argument of the Case (4) applied on the domino corners $A$ and $C$ gives that $\alpha \stackrel{p_{r}}{\sim} \sigma$. On the other hand if $C$ is a horizontal domino corner then the argument of the Cases (1),(2) and (3) applied on $A$ and $C$ gives $\alpha \stackrel{p_{r}}{\sim} \sigma$. Therefore $\alpha \stackrel{p_{r}}{\sim} \beta$. On the other hand $T$ has a corner, say $C^{\prime}$, lying in $(T, B$, ne) one can use the same argument in the transpose of $T$.

If there no domino corner in $T$ other then $A$ and $B$ there are two possibility as illustrated in Figure $5(\mathrm{~b})$ and Figure 5 (c). Observe that the case given in Figure 5 (b) is just the transpose of the Case (4) illustrated in Figure 4(d), therefore it follows directly that $\alpha \stackrel{p_{r}}{\sim} \beta$.

For the latter case shown in Figure 5 (c), observe that the shaded area is a $r$-staircase shape and we must have either the domino corner $A$ or $B$ of $T$ labeled by $(n, n)$. Here we assume $A$ is labeled by $(n, n)$ since for the other case one can use the same argument on the transpose tableau $T^{t}$. So as Figure 5 (c) illustrates, let $x_{1} \ldots x_{k}$ be the labels of horizontal domino cells and $y_{1} \ldots y_{l}$ be the vertical domino cells which are both positive decreasing sequence such that $r+1=k+l$. Observe that $\eta\left(T^{\uparrow A}\right)=\eta\left(T^{\uparrow B}\right)=x_{k}>0$ therefore $\alpha_{n}=\beta_{n}=x_{k}$. Let $\tilde{u}$ be a signed word which is a shuffle of $x_{1} \ldots x_{k-1}$ and $-y_{1} \ldots-$ $y_{l}$. It is easy to see that $P^{r}\left(n \tilde{u} x_{k}\right)=T=P^{r}\left(-n \tilde{u} x_{k}\right)$, and $n \tilde{u} x_{k} \stackrel{\mathrm{D}_{3}^{r}}{\sim}(-n) \tilde{u} x_{k}$. On the other hand $P^{r}(n \tilde{u})=T^{\uparrow A}$ and $P^{r}(-n \tilde{u})=T^{\uparrow B}$ and by induction hypothesis we have $n \tilde{u} \stackrel{p_{r}}{\sim} \alpha_{1} \ldots \alpha_{n-1}$ and $(-n) \tilde{u} \stackrel{p_{r}}{\sim} \beta_{1} \ldots \beta_{n-1}$. Hence $\alpha=\alpha_{1} \ldots \alpha_{n-1} x_{k} \stackrel{p_{r}}{\sim} n \tilde{u} x_{k} \stackrel{D_{3}^{r}}{\sim}(-n) \tilde{u} x_{k} \stackrel{p_{r}}{\sim} \beta_{1} \ldots \beta_{n-1} x_{k}=\beta$.

\section{Acknowledgments}

The author is thankful to Nantel Bergeron, Huilan Li and Victor Reiner for helpful discussions and comments.

\section{References}

[1] D. Barbash, D. Vogan, Primitive ideals and orbital integrals in complex classical groups, Math. Ann., 259, (1982), 153-199.

[2] C. Bonnafé, On Kazhdan-Lusztig cells in type B. arXiv:0806.0214v1.

[3] C. Bonnafe, M. Geck, L. Iancu, T.Lam, On the insertion and Kazhdan-Lusztig cells in type $B_{n}$, math.RT/0609279. Progress in Math (Lusztig Birthday Volume), Birkhauser, to appear.

[4] D. Garfinkle, On the classification of primitive ideals for complex classical Lie algebras I, Compositio Mathematica 75 (1990), 135-169.

[5] D. Garfinkle, On the classification of primitive ideals for complex classical Lie algebras II, Compositio Mathematica $\underline{8} 1$ (1992), 307-336.

[6] D. Garfinkle, On the classification of primitive ideals for complex classical Lie algebras III, Compositio Mathematica $\underline{8} 8$ (1993), 187-234. 
[7] M. Geck, Left cells and constructible representations, Representation Theory 9, (2005), 385-416.

[8] I. G. Gordon, Quiver varieties, category $\mathcal{O}$ for rational Cherednik algebras, and Hecke algebras, math.RT/0703153

[9] I.G. Gordon, M. Martino, Calogero-Moser spaces, reduced rational Cherednik algebras, and two sided cells, math.RT/0703150.

[10] A. Joseph, On the variety of a highest weight module, J. of Algebra, $\underline{8} 8$, (1984), 238-278.

[11] D. Kazhdan, G. Lusztig, Representations of Coxeter groups and Hecke algebras, Invent. Math., 53 (1979), 165-184.

[12] D. E. Knuth, Permutations, matrices and generalized Young tableaux, Pacific J. Math., 34 (1970), 709-727.

[13] , T. Lam, Growth diagrams, domino insertion, and sign-imbalance, J. Comb. Theory Ser. A., 107 (2004), 87-115.

[14] M.A.A. van Leeuwen, The Robinson-Schensted and Schützenberger algorithms, an elementary approach, Electronic J. of Combin. $\underline{3}$ (1996).

[15] G. Lusztig, Hecke algebras with unequal parameters, CRM Monographs Ser.18, Amer.Math.Soc. (2003), 136p.

[16] W. McGovern, Left cells and domino tableaux in classical Weyl groups, Composito Mathematica, 101 (1996), 77-98.

[17] T. Pietraho, Knuth Relations for the Hyperoctahedral groups, arXiv:0803.3335.

[18] C. Schensted, Longest increasing and decreasing subsequences, Canad. J. Math., 13, (1961), 179191.

[19] M. P. Schützenberger, La correspondence de Robinson, Combinatoire et Représentation du Groupe Symétrique, Lecture Notes in Math.,79, Springer, Berlin (1977), 59-135.

[20] M.Taskin, Plactic relations for $r$-domino tableaux, arXiv:0803.1148.

[21] M. Shimozono, D. White, A color-to-spin domino Schensted algorithm. Electronic J. of Combinatorics, $\underline{8}$, no. 1, (2001), Research Paper 21, 50 pp.

[22] D. Vogan, Ordering of the primitive spectrum of a semisimple Lie algebra, Math. Ann. 248 (1980), 195-2003. 